\title{
A systematic review of dietary behaviour change interventions in socioeconomically disadvantaged adolescents: theoretical and practical approaches
}

\author{
S. Bel-Serrat ${ }^{1}$, E. Greene ${ }^{1}$, A. Mullee ${ }^{2}$ and C.M. Murrin ${ }^{1}$ \\ ${ }^{1}$ National Nutrition Surveillance Centre, School of Public Health, Physiotherapy and Sports Science, University \\ College Dublin, Dublin, Ireland and \\ ${ }^{2}$ Department of Health and Nutritional Sciences, Institute of Technology Sligo, Sligo, Ireland.
}

Less affluent adolescents show unhealthier dietary behaviours than their more affluent peers ${ }^{(1)}$. Eating habits are established during adolescence and tend to track into adulthood ${ }^{(2,3)}$. Addressing adolescents' unhealthy behaviours will contribute not only to their future health, but to the health of the next generations ${ }^{(4)}$. However, there is a lack of evidence on the strategies used to change dietary behaviour in socioeconomically disadvantaged adolescents and on the effectiveness of these strategies. This review aims to provide a synthesis of nutrition interventions used in this population group and to inform the development of future intervention studies that seek to promote healthier diets among these adolescents.

Five electronic databases (PubMed, Web of Science, CINAHL, PsycINFO and ERIC) were searched from 2013 to November 2020. The search strategy used the following set of descriptors: nutrition and lifestyle interventions, health promotion, adolescents, and behaviour change strategies. Included studies met the following criteria: 1) sample comprised of low-income individuals aged 12-18 years, 2) reported on dietary and/or lifestyle, including diet, interventions, 3) conducted in urban settings of high income countries, 4) were preventive trials (intervention studies) for healthy individuals including randomized controlled trials, quasi-experimental trials, and pre-post studies, 5) reported quantitative findings on intervention dietary components, and 6) published in peer-reviewed journals.

Forty-six manuscripts (38 intervention studies) were included. The main reasons for exclusion of full texts $(n=163)$ were not targeting adolescents, sample income level unknown, not targeting a low-income sample, and lack of quantitative results on intervention dietary outcomes. Studies were classified by intervention type as those focusing on: hedonic determinants of dietary intake $(n=1)$, environmental changes to promote a specific dietary intake $(n=3)$, cognitive determinants $(n=29)$, and multicomponent strategies $(\mathrm{n}=13)$. The Social Cognitive Theory was the most commonly applied theoretical framework, either alone or combined with other theoretical frameworks. A variety of successful intervention strategies in achieving positive dietary behaviour changes were identified; however, follow-up periods tended to be short and those interventions with longer ones did not always observe sustained benefits. Most of the intervention studies targeted multiple dietary outcomes and success was not observed across all the outcomes.

Despite the heterogeneity of the studies, long-term theory-driven interventions targeting one single dietary factor seem more promising in obtaining sustained dietary behaviour changes. More nutrition programs examining the long-term benefits and sustainability of the interventions are needed to inform future policies tailored to disadvantaged adolescents.

\section{References}

1. WHO Regional Office for Europe (2020) 2017/2018 Health Behaviour in School-Aged Children survey in Europe and Canada. International report.

2. Ambrosini GL, et al. (2014) Obesity 22(2), 458-65.

3. te Velde SJ, et al. (2007) Br J Nutr 98(2), 431-8.

4. Sawyer SM, et al. (2012) The Lancet 379, 1630-1640. 